\title{
Apprentissage
}

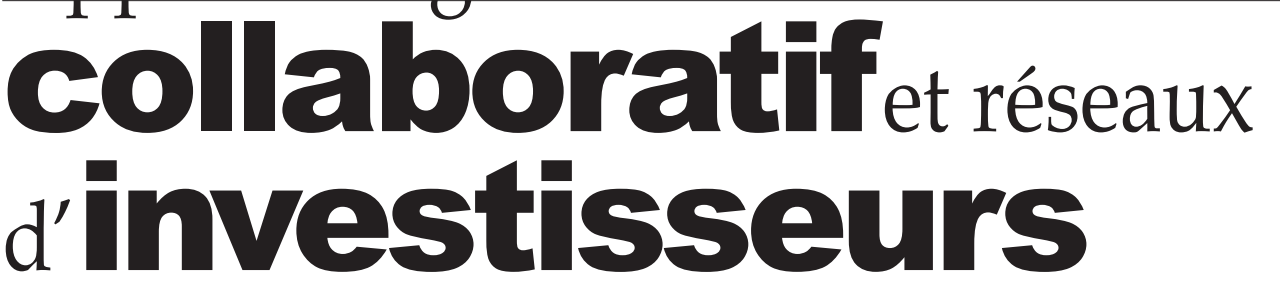

\section{en capital-risque}

L'investissement en capital risque suppose une évaluation de l'incertain. Cette dernière s'appuie sur des informations tacites et informelles qui font du lien social un vecteur important de leur obtention. La relation contractuelle devient la condition formelle d'un apprentissage collaboratif informel qui permet la création de liens sociaux entre

I'entrepreneur et ses investisseurs. Ces derniers utilisent le cadre légal des syndications comme un lieu d'échanges informels, réciproques et différés de ressources dont la logique relève de la théorie du don.

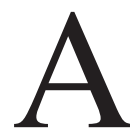
ux États-Unis, l'industrie du capital-risque finance la création des entreprises à fort taux de croissance (les « start-up »). Elle est régulièrement identifiée comme un facteur déterminant de la dynamique d'innovation et de développement de nouveaux secteurs industriels (semi-conducteurs, internet, biotechnologies, etc.). Les sociétés de capital-risque (SCR) financent les entreprises à travers une série d'investissements séquentiels. Lorsqu'une entreprise a utilisé les ressources financières d'un tour de financement, elle sollicite ses investisseurs pour un nouveau tour dans le cadre d'une augmentation de capital. Le financement d'une entreprise entre sa création et son introduction en Bourse ou son rachat par une grande entreprise se réalise à travers quatre tours (Seed, Early, Expansion et Later stage). Il convient de distinguer le premier tour (Seed Stage) qui correspond au financement de la création de l'entreprise, des autres tours qui correspondent au financement du développement de l'entreprise. Les tours d'investissement correspondent à une maturation de l'entreprise et, corollairement, à une décroissance du risque. 
Les SCR financent une même entreprise à travers des syndications qui regroupent plusieurs investisseurs pour réaliser un tour de financement. Une syndication est composée d'une SCR « chef de file », qui est la SCR à l'origine de la relation avec l'entreprise financée, ses représentants siègent au conseil d'administration, ils sont très impliqués dans la gestion de l'entreprise et nourrissent des liens sociaux forts ${ }^{1}$ avec l'entrepreneur. Par convention tacite, la SCR chef de file invite d'autres investisseurs à coinvestir dans sa syndication. Ces derniers sont des investisseurs passifs car ils ne sont pas impliqués dans la gestion de l'entreprise financée. Toutes les SCR peuvent être chef de file ou investisseur passif selon qu'elles sont ou non à l'origine de la syndication. La problématique réticulaire vise à analyser pourquoi et comment se créent et se cristallisent dans des syndications des réseaux d'investisseurs en capital-risque.

Traditionnellement, l'analyse des syndications relève de la théorie financière. Cette dernière appréhende les syndications à l'aune des principes de la diversification des investissements pour gérer les risques financiers. Dans cette perspective, les SCR, par l'intermédiaire des syndications, partagent entre elles des informations et leur expertise pour réduire le risque tout en le diversifiant (Gompers et Lerner, 2001). Ainsi, plus le risque est important et plus les «capital-risqueurs» cherchent à le diversifier en invitant d'autres SCR dans leurs syndications (Bygrave, 1987; Gomper et Lerner, 1998). À l'inverse, lorsque le risque se réduit, le nombre de capital-risqueurs est censé diminuer. Selon cette logique, le nombre de SCR devrait être le plus important dans la syndication du premier tour (seed) car le risque est maximal et être le plus faible au dernier (later stage) lorsque le risque est moindre en raison de la plus grande maturité de l'entreprise. L'analyse empirique invalide ces hypothèses. Concernant les 2715 syndications réalisées aux États-Unis en 2003, sur les 167 premiers tours d'investissement, 92 (soit 55,09\%) ne sont constitués que par un seul investisseur et 46 (soit 27,54\%) correspondent à une syndication de deux investisseurs. Seules 29 syndications (soit $17,37 \%$ ) sont constituées de trois investisseurs ou plus. De plus, le nombre moyen d'investisseurs augmente avec la maturité de l'entreprise. Il est de 1,68 au premier tour, de 2,34 au second, de 2,8 au troisième et de 3,38 au dernier tour.

Notre recherche vise à analyser les syndications à l'aune de la théorie des ressources (Penrose, 1959) pour montrer qu'elles matérialisent des réseaux de partage de ressources entre acteurs interdépendants. Dans cette perspective, les syndications sont hétérogènes. Outre les SCR, les syndications sont également composées de grandes entreprises (Intel, Lucent Technologies, Siemens, etc.), de banques d'affaires (JP Morgan, Goldman Sachs, etc.) et de sociétés de capital-investissement (Carlyle Group, Bain Capital, etc.). Les investisseurs s'invitent informellement dans les syndications pour partager leurs ressources.

1. Granovetter (1973) définit la force d'un lien comme étant « une combinaison (probablement linéaire) de la quantité de temps, de l'intensité émotionnelle, de l'intimité (la confiance mutuelle) et des services réciproques qui caractérisent ce lien». 
La compréhension des réseaux d'investisseurs matérialisés par les syndications de capital-risque rend nécessaire l'analyse du lien entre les investisseurs et les créateurs d'entreprises pour ensuite appréhender la nature des échanges entre les investisseurs. Dans une première partie, nous exposerons la spécificité du premier tour d'investissement (Seed) pour montrer qu'il ne relève pas de la gestion du risque mais de la gestion de l'incertain (Knight, 1921). Le premier tour d'investissement peut être considéré comme un dispositif contractuel d'apprentissage collaboratif permettant à un investisseur de créer un lien social fort avec le créateur d'entreprise afin d'obtenir les informations nécessaires à la réduction de l'incertain. Dans une seconde partie, nous analyserons en quoi la position de « chef de file » permet à une SCR d'inviter dans ses syndications les partenaires nécessaires au développement des entreprises dans lesquelles elle a investi (grandes entreprises, banques d'affaires et sociétés de capital-investissement) en utilisant son lien social fort avec le créateur d'entreprise comme une ressource à offrir pour initier l'échange avec les autres investisseurs. Nous montrerons que ces échanges de ressources à travers des invitations réciproques, informelles et différées dans le cadre des syndications relèvent de la théorie de l'échange par le don (Mauss, 1930).

\section{I. - LE RÔLE DES RÉSEAUX SOCIAUX DANS L'ÉVALUATION DES PROJETS D'INVESTISSEMENT}

\section{Risque et incertain dans l'activité de capital-risque}

La diversification des risques s'appuie sur le présupposé implicite que les risques sont probabilisables. Les travaux de Knight (1921) ont montré qu'il existe en fait deux natures d'incertitude, celle qui est mesurable: le risque, et celle qui ne l'est pas: l'incertain. La différence pratique entre ces deux catégories est que les investissements

\section{MÉTHODOLOGIE}

Cette recherche s'appuie sur une douzaine d'entretiens semi-directifs avec des investisseurs en capital-risque américains représentant les quatre catégories d'investisseurs (Sequoia Capital, Kleiner-Perkins-Caufield\&Byers-KPCB, Atlas Venture, Partech, Sofinnova, Ridge Venture, Lucent Venture Partners, Innovacom, Sprout, TH Lee, KPN Venture et Intel Capital) pour explorer leurs relations avec les entrepreneurs et leurs relations avec les autres investisseurs. L'analyse quantitative des syndications s'appuie sur la base de données MoneyTree de 2003, gracieusement fournie par PriceWaterCoopers qui regroupe les 2715 syndications réalisées aux États-Unis cette année-là. Pour chaque syndication, les informations disponibles sont le nom et le secteur de l'entreprise financée, le tour d'investissement (Seed, Early, Expansion ou Later), le montant investi et le nom des investisseurs. La base de données a été complétée pour définir le type d'investisseur (SCR, groupe industriel, banque d'affaires ou société de capital-investissement) à partir du Galante's Venture Capital \& Private Equity Directory, gracieusement fournie par Down Jones. 
qui représentent un risque correspondent à des classes d'actifs suffisamment nombreuses et ayant suffisamment de données historiques pour qu'une probabilisation de la rentabilité et de la possibilité de défaillance soient possibles. En revanche, un actif sera jugé incertain (et donc nonprobabilisable) quand il correspond à une catégorie d'actif unique et qu'il n'a pas d'historique permettant de déployer des outils d'analyse financière.

La théorie de la diversification des investissements appliquée aux activités de capitalrisque présuppose que les entreprises de forte croissance en période de création constituent des risques pouvant être gérés comme tels. Cependant, avant la création de l'entreprise, qui accompagne ou précède de peu le premier tour, cette dernière n'a ni produit, ni client, ni chiffre d'affaires et bien souvent les créateurs de l'entreprise n'ont aucune expérience entrepreneuriale. Lorsque l'innovation technologique est une innovation radicale, il est difficile d'évaluer objectivement le potentiel et la fiabilité de cette technologie. À ce stade, il y a peu d'informations explicites permettant de déployer les outils classiques de l'analyse financière et de la gestion des risques. Un investissement au capital d'une entreprise au moment de sa création correspond à un investissement incertain. Cela suppose des pratiques de gestion différentes notamment en matière de collecte de l'information nécessaire à l'évaluation de l'incertitude.

Pour gérer l'incertain relatif au premier tour d'investissement, le capital-risqueur va collecter des informations pour évaluer trois sources d'incertitude: le marché, le produit et l'entrepreneur. La dernière dimension qui concerne les compétences, la personnalité et les capacités managériales du créateur d'entreprise est souvent présentée par les capitalrisqueurs comment étant la plus importante. Ces informations sont rarement publiques et explicites. L'évaluation du risque s'appuie sur des connaissances explicites alors que l'évaluation de l'incertain s'appuie sur des connaissances tacites et diffuses. La circulation des connaissances tacites est fortement dépendante des interactions interindividuelles (Nonaka, 1994). Par nature, les connaissances tacites sont informelles, peu commercialisables et fortement personnalisées au sens où leur valeur est plus liée à la qualité de l'émetteur de l'information qu'à l'information elle-même. La dimension tacite de la connaissance nécessaire à l'évaluation de l'incertain confère une grande importance à la nature du lien social entre le détenteur de la connaissance tacite et l'investisseur qui utilise cette connaissance pour évaluer l'incertain.

Le capital-risqueur conduit l'entrepreneur à expliciter son projet afin de l'évaluer. Leurs interactions génèrent une création de connaissances car le capital-risqueur va amener l'entrepreneur à se projeter dans des dimensions qu'il n'avait pas explicité comme le potentiel du marché ou la protection légale du produit. Quand bien même ces informations sont explicitées et formalisées, notamment dans le business plan, elles restent des projections dont la faisabilité est subjectivement perçue. Les capitalrisqueurs sont dans une situation d'évaluation subjective de l'incertain. Ils se forgent des convictions sur des bribes d'informations. C'est en rencontrant plusieurs fois l'entrepreneur que le capital-risqueur se construit une certitude sur ses compétences et sa personnalité. 


\section{Le rôle des réseaux sociaux dans la réduction de l'incertain}

Dans les activités de capital-risque, l'essentiel de l'évaluation est réalisé lors du premier tour. En moyenne, un capital-risqueur reçoit 500 business plans par an. $90 \%$ des dossiers sont rapidement rejetés et sur les $10 \%$ restants, seuls 5 ou 6 investissements seront réalisés - soit $1 \%$ des propositions. Les capital-risqueurs vont utiliser leurs réseaux sociaux pour collecter les informations tacites nécessaires à la réduction de l'incertain. Ils vont contacter des ingénieurs et des professeurs d'université pour valider la technologie, ils vont contacter des cadres d'entreprises pour évaluer le potentiel du marché et vont contacter des amis, des relations de travail, des anciens collègues ou des anciens professeurs pour évaluer les entrepreneurs. La collecte de ces informations se fait de manière informelle et hors de toute relation marchande contractuelle. Un capital-risqueur californien d'Atlas Venture illustre cette démarche:

«Quand j'ai reçu le business plan de Fireclick qui m'avait été recommandé par un dirigeant d'entreprise que je connais, pour valider leur modèle économique j'ai appelé un ami qui travail à la direction du marketing d'Amazon et un autre qui est directeur technique chez DoubleClick. Pour valider leur algorithme d'accélération d'affichage des pages internet, je me suis adressé à un professeur d'informatique que j'avais eu comme enseignant à l'université de Stanford ».

Le caractère tacite de l'information met les réseaux sociaux au centre de l'évaluation de l'incertain par le capital-risqueur et font du lien social un vecteur d'obtention d'information supérieur aux strictes relations marchandes (Granovetter, 2005).
La qualité de l'évaluation de l'incertitude va dépendre de la qualité des réseaux sociaux des capital-risqueurs et de leur degré d'encastrement social avec les réseaux des entrepreneurs qu'ils évaluent (Ferrary, 2003). La qualité de l'évaluation ne dépendra pas de liens sociaux homophiles avec d'autres SCR mais de liens sociaux hétérophiles avec différents acteurs économiques. De ce fait, les capital-risqueurs entretiennent une multitude de liens faibles hétérophiles pourvoyeurs d'informations nécessaires à la réduction de l'incertitude. La circulation de l'information tacite relative au projet de création d'entreprise et à l'entrepreneur dépend du degré d'encastrement du capital-risqueur et de l'entrepreneur dans les mêmes réseaux sociaux. Certains capital-risqueurs préfèrent renoncer à un investissement lorsque l'absence d'interconnexion entre son réseau et celui de l'entrepreneur ne lui permet pas de réaliser une prise de référence. Ainsi, un capital-risqueur d'expliquer:

«Une fois, j'ai analysé un dossier prometteur sur le plan de la technologie et des perspectives de marché mais je n'ai pas réussi à obtenir des informations sur l'entrepreneur. Personne ne le connaissait dans mes relations. Dans le doute, j'ai préféré renoncer à investir car ne pas avoir d'information représente un risque trop important. »

Avant de considérer un projet, un capitalrisqueur n'a souvent aucun lien social direct avec l'entrepreneur, la mise en relation est souvent le fait d'un intermédiaire de confiance. Dans le processus d'évaluation, un lien faible se crée entre les deux acteurs. En devenant actionnaire et en siégeant au conseil d'administration de l'entreprise, la SCR s'implique fortement dans la gestion 
de l'entreprise. La fréquence des interactions entre le capital-risqueur et le créateur d'entreprise contribue à la création d'un lien fort entre ces deux personnes et permet une circulation des informations tacites relatives à la personnalité, aux compétences managériales et commerciales de l'entrepreneur. Le premier tour d'investissement constitue une situation légale et organisationnelle permettant un apprentissage mutuel par la collaboration entre l'entrepreneur et le capital-risqueur. Cet apprentissage permet la création d'un lien social fort et d'une relation de confiance pourvoyeuse d'informations nécessaires à l'évaluation de l'incertain. Face à une situation incertaine dans laquelle le lien social n'existe pas pour transmettre l'information, les acteurs utilisent le contrat pour favoriser la socialisation. La contractualisation de l'investissement devient un moyen d'encastrement social et de création du lien social. Un capital-risqueur de Kleiner, Perkins, Caufield \& Byers illustre cette relation:

« Je m'implique beaucoup dans le management d'une entreprise dans laquelle nous avons investi et pour lesquelles j' appartiens au conseil d'administration. Je donne beaucoup de conseils. J'interviens dans les recrutements, notamment en faisant jouer notre réputation pour convaincre les meilleures personnes. Je facilite les contacts avec des grandes entreprises. Ainsi, j'ai mis en relation avec American Express une de nos start-up qui développe une activité liée aux moyens de paiement. La direction de cette dernière n'aurait pas répondu si je n'étais pas intervenu... quand la situation le nécessite, je peux être en relation quotidienne avec l'entrepreneur. Parfois je m'installe à plein temps dans ses locaux pour mieux travailler avec lui. »
Ce processus d'apprentissage correspond à un modèle à deux périodes de la théorie de l'agence. À la première période, il y a une asymétrie entre le principal (SCR) et l'agent (l'entrepreneur). Le premier tour d'investissement va initier un processus d'apprentissage de l'entrepreneur par la SCR. L'investisseur va pouvoir se rendre compte si l'entrepreneur est rigoureux et sait manager, si le développement technologique se déroule selon le planning prévu et si les clients sont intéressés par le prototype. Il y a une réduction de l'incertain par un processus d'apprentissage induit par l'implication dans gestion de l'entreprise. Au cours du premier tour, l'investissement évolue d'une nature incertaine à une nature risquée. De ce fait, la SCR devient un « investisseur privilégié » en raison de sa détention d'informations privées que ne possèdent pas des acteurs extérieurs. Elle sera de ce fait la mieux à même de juger de la viabilité réelle du projet pour éventuellement investir dans un second tour de financement ou au contraire y renoncer.

En raison de la contrainte de socialisation particulièrement forte au premier tour, l'hypothèse peut être faite que peu de SCR seront impliquées dans ce tour car un même entrepreneur ne peut pas construire plusieurs liens forts avec plusieurs SCR et, similairement, une SCR ne peut pas construire des liens forts avec une multitude d'entreprises en phase de création. La création d'un lien social et son entretien représentent un coût caché d'autant plus élevé que ce lien est fort. Cette contrainte liée à la ressource temporelle limitée des acteurs explique pourquoi 55,09\% des syndications de premier tour de notre population ne sont composées que d'un seul investisseur et $27,54 \%$ de deux. 
La construction du lien social constitue un investissement «caché » que les SCR chercheront à rentabiliser en favorisant le développement des entreprises qu'elles ont financé. En détenant des informations privées, les SCR chef de file possèdent des ressources recherchées par d'autres acteurs économiques qui n'ont ni le temps ni la compétence pour les obtenir.

\section{II. - POUR UNE THÉORIE DE L'ÉCHANGE DANS LES RÉSEAUX D'INVESTISSEURS EN CAPITAL-RISQUE}

\section{Ressources et objectifs \\ des investisseurs en capital-risque}

Aux États-Unis, la participation aux syndications d'investissement n'est pas réservée aux sociétés de capital-risque. L'analyse des 2715 syndications réalisées en 2003 montre que 1382 investisseurs y ont participé: 790 SCR (57,29\% des investisseurs); 322 sociétés de capital-investissement (23,35\%); 182 groupes industriels $(13,2 \%)$ et 85 banques $(6,16 \%)$. La nature des ressources détenues par un acteur est déterminante à double titre dans la dynamique des réseaux. D'une part, elle est à l'origine de l'attraction de partenaires qui souhaitent créer le lien pour accéder à la ressource et, d'autre part, la dépendance de l'acteur à l'égard d'autres ressources va déterminer sa stratégie de construction de liens (Tsai, 2000). L'exposé de l'hétérogénéité des ressources et des objectifs des investisseurs en capital-risque permet de comprendre la nature de leur interdépendance.

\section{Les sociétés de capital-risque}

La performance financière d'une SCR dépend des plus-values qu'elle réalise en revendant ses participations. Cela peut résulter soit d'une introduction en Bourse, soit d'un rachat des entreprises financées. Le succès d'une SCR dépendant de sa capacité à mettre en relation les entreprises qu'elle finance avec des grands groupes industriels, des banques d'affaires et/ou des sociétés de capital-investissement. Les ressources que détient une SCR et qui intéressent ses partenaires sont, d'une part, les informations privées concernant les startup dont elle a financé le premier tour et d'autre part, sa prérogative en tant que chef de file de la syndication pour inviter des investisseurs.

\section{Les grandes entreprises}

Leur objectif est d'améliorer leur portefeuille de technologies, notamment en acquérant des start-up ayant développé des technologies complémentaires. L'enjeu est d'obtenir des informations sur ces entreprises préalablement à une éventuelle acquisition. Or, aux États-Unis, lorsqu'une entreprise est non-côtée en Bourse, ses obligations de publication d'informations sont extrêmement réduites. L'intérêt pour un grand groupe industriel à participer à une syndication est d'accéder à des informations privées avant ses concurrents. Ces grandes entreprises détiennent des ressources qui peuvent favoriser le développement des start-up (partenariats technologiques, accords commerciaux, etc.) et elles peuvent les racheter lorsqu'elles développent des technologies complémentaires.

\section{Les banques d'affaires}

Leur objectif est de trouver des sources d'activités pour leurs compétences d'organisation d'acquisition d'entreprise et d'introduction en Bourse. En participant à des 
syndications, elles accèdent à des informations privées qui leurs permettent d'être les mieux à même de préparer une introduction en Bourse ou une acquisition et de nouer des liens privilégiés avec de potentiels futurs clients.

\section{Les sociétés de capital-investissement}

Elles financent leurs acquisitions par endettement (Leverage Buy Out). Cela suppose que les entreprises qu'elles acquièrent génèrent des profits ou qu'une rationalisation de leur gestion permette d'en dégager suffisamment pour rembourser l'endettement induit par leur rachat. Les start-up matures qui génèrent des profits et qui ont des besoins financiers auxquels ne peut faire face une SCR constituent une possibilité d'investissement pour ces sociétés. La participation à une syndication permet d'accéder à des informations privées relatives à l'entreprise financée pour éventuellement l'acquérir ultérieurement.

\section{La dynamique d'échange entre investisseurs interdépendants}

Il y a une division du travail au sein de la communauté des investisseurs dans laquelle le rôle des SCR est de convertir un projet incertain en un projet risqué par la collecte d'informations tacites et/ou privées. Au premier tour d'investissement, seuls les SCR participent au financement des entreprises car elles sont les seules à détenir cette compétence de conversion de l'incertain en risque. L'analyse des 167 syndications de premier tour d'investissement montre que sur les 243 investisseurs impliqués, 211 sont des SCR (86,5\% des investisseurs), 14 sont des sociétés de capitalinvestissement (6\%), 14 sont des groupes industriels $(6 \%)$ et 4 sont des banques
(1,5\%). À titre d'exemple, en 2003, si l'on considère Intel, qui a réalisé 43 investissements dans des syndications, JP Morgan, qui en a réalisé 40 et Bain Capital, Carlyle Group et Thomas Lee, trois des plus importants sociétés américaines de capital-investissement qui ont réalisé 34 investissements; aucuns n'ont participé à une syndication de premier tour.

Pour le financement des tours d'investissement suivants celui dédié à la création de l'entreprise, les SCR vont user de leur prérogative informelle de " chef de file de la syndication » pour inviter des investisseurs détenant des ressources nécessaires au développement de l'entreprise et préparer leur stratégie de sortie. Ces nouvelles catégories d'investisseurs acceptent de participer aux syndications principalement d'avant-derniers et de derniers tours (Expansion et Later Stage). On constate une diversification des investisseurs avec la maturation des entreprises. Au dernier tour, les sociétés de capital-investissement représentent $17,04 \%$ des investisseurs, les grandes entreprises $10,1 \%$ les banques d'affaires 8,38\% et les SCR 64,48\%.

$\mathrm{Du}$ fait de leur interdépendance dans un environnement incertain, les investisseurs vont utiliser des dispositifs contractuels pour instituer les conditions de création de lien social permettant la circulation d'informations tacites et le partage de ressources. En participant à une syndication, les investisseurs peuvent interagir avec les entrepreneurs et avoir des informations privées. Ils bénéficient d'un avantage informationnel par rapport à leurs concurrents. Les capital-risqueurs sont conscients de ce besoin de création de lien social pour la circulation de l'information. Il est dans l'intérêt de la SCR d'inviter une grande entreprise dans une syn- 
dication pour lui permettre d'accéder par la création d'un lien social aux informations tacites et/ou privées. Cette invitation ne se fera pas au premier tour mais dans les syndications des tours suivants quand l'incertitude aura été fortement réduite par l'expérience accumulée par la SCR chef de file. Les syndications des tours ultérieurs sont utilisées comme un moyen pour créer un lien faible entre les nouveaux investisseurs et l'entreprise financée qui permettra la circulation réticulaire d'informations et de ressources. Ce qui se joue dans la composition d'une syndication est moins le financement des entreprises que la création de relations sociales entre acteurs interdépendants pour apporter les ressources à l'entreprise financée.

\section{La théorie du don pour expliquer des échanges réciproques, informels et différés entre les investisseurs}

Formellement et contractuellement, il y a un transfert de ressources financières entre les investisseurs et l'entreprise financée. Cependant, de manière informelle, des engagements tacites sont pris pour des contributions futures non-contractualisées. Les relations entre les participants des syndications sont informelles. Aucun contrat ne régit les relations entre les investisseurs. Le contrat formel est conclu entre l'entreprise et les investisseurs. Ainsi, aucune contrainte légale n'oblige une banque d'affaires à organiser ultérieurement l'introduction en Bourse de l'entreprise financée par la syndication à laquelle elle a participé. De même, une grande entreprise n'a aucune obligation légale de racheter l'entreprise qu'elle a contribué à financer par sa participation à la syndication. Cette réciprocité est une norme de l'échange qui résulte d'une convention informelle. De plus, cette réciprocité est par nature différée. La banque d'affaires réalisera l'introduction en Bourse et la grande entreprise ne rachètera éventuellement la start-up que plusieurs mois, voire plusieurs années après avoir été invitées à une syndication. Ces caractéristiques de l'échange (informel et différé) sont celles de l'échange par le don décrit par Mauss (1930), selon lequel un agent doit d'abord donner pour recevoir, qu'accepter un don suppose l'acceptation de l'obligation de rendre et que ne pas rendre entraîne la cessation des échanges. Ces normes de l'échange sont informelles et leur respect est conditionné par la coercition sociale qu'exerce la communauté. Ces normes expliquent les échanges implicites qui se nouent entre les investisseurs au sein des syndications. La SCR chef de file utilise sa position d' «investisseur privilégié» comme une ressource dans ses échanges avec les autres investisseurs. L'invitation d'une SCR est un don accordé à une banque, un groupe industriel ou une société de capital-investissement qui sera rendu ultérieurement par un rachat ou une introduction en Bourse qui constituera le contredon. Ainsi, la SCR Sequoia Capital invite régulièrement Cisco Systems à participer aux syndications de financement de startup du secteur de l'équipement en télécommunication. Entre 1993 et 2002, Cisco Systems a racheté 10 des 19 entreprises d'équipement en télécommunication revendues par Sequoia Capital. Un associé de Sequoia Capital décrit cette relation:

«Sequoia Capital et Cisco Systems entretiennent des liens privilégiés. Sequoia Capital a financé Cisco lors de sa création et plusieurs associés de Sequoia se sont impliqués dans la gestion de Cisco. Don Valentine, un associé de Sequoia est vice- 
chairman de Cisco. On connaît bien leur stratégie et leurs technologies. Nous investissons dans des start-up en gardant à l'esprit nos liens privilégiés avec Cisco. Quand on prépare un nouveau tour d'investissement pour une entreprise qui peut intéresser Cisco, nous invitons cette dernière dans notre syndication. Cela aide pour des partenariats technologiques et commerciaux, puis éventuellement, pour un rachat. »

L'encastrement des investisseurs dans un réseaux leur offre des opportunités d'accession à des ressources complémentaires aux leurs mais constitue également une contrainte qui est l'obligation à la réciprocité sous peine d'exclusion des échanges au sein du réseau. Ainsi, une grande entreprise qui utiliserait des informations privées obtenues par sa participation à une syndication pour développer en interne une technologie concurrente à celle de la start-up qu'elle contribue à financer et donc ne la rachèterait pas à terme, ne se conformerait pas à son obligation de réciprocité. De ce fait, elle serait exclue des syndications futures et sa réputation au sein de la communauté des investisseurs serait détériorée, réduisant d'autant sa probabilité d'être à nouveau invitée.

L'échange par le don est le principe d'échange entre les investisseurs en capitalrisque et c'est la densité des réseaux sociaux que constituent ces investisseurs qui sécurise cette forme d'échange en créant une contrainte socio-économique à respecter la norme de réciprocité. L'encastrement social modifie l'horizon social et temporel d'optimisation des acteurs (Ferrary et Pesqueux, 2004). Plus cet encastrement sera important et plus les acteurs auront intérêt à respecter la convention de l'échange par le don pour préserver leurs gains futurs.

\section{CONCLUSION}

La fonction économique des SCR ne se limite pas au financement de la création des start-up. Elle consiste également à collecter des informations sur les projets d'entreprise grâce à leurs liens faibles hétérophiles avec une multitude d'acteurs économiques et d'en co-créer grâce à la construction d'un lien fort avec l'entrepreneur pour convertir une incertitude incertaine en une incertitude risquée. En invitant des organisations différentes à leurs syndications, les SCR chef de file leurs donnent un accès à des informations privées qui les rendront capables de prendre des décisions plus efficientes que leurs concurrents tout en permettant à ces SCR de mobiliser les ressources nécessaires au développement des entreprises qu'elles financent. La participation à une syndication induit un processus d'apprentissage entre les investisseurs et l'entreprise financée qui modifie le processus des décisions futures et crée une asymétrie d'information entre les « insiders » et les « outsiders ». La syndication constitue une condition contractuelle de création du lien social nécessaire à la diffusion des informations tacites et/ou privées.

La sociologie économique montre en quoi la socialisation ex-ante des échanges économiques permet leur réalisation. Dans cette recherche, l'objectif était de montrer comment des acteurs économiques désocialisés mais conscients de l'importance du lien social pour véhiculer des connaissances tacites et/ou privées développent les conditions contractuelles de création de ce lien social, en l'occurrence la syndication. Si l'analyse des réseaux a mis en évidence dans de nombreux secteurs économiques le rôle du lien social dans la conclusion du 
contrat marchand, elle omet bien souvent d'expliquer la genèse de cette socialisation. D'un point de vue théorique, l'analyse des réseaux d'investisseurs en capital-risque permet d'explorer une perspective nouvelle de la relation entre le contrat marchand et le lien social. Les investisseurs utilisent le contrat de financement comme un dispositif formel d'apprentissage collaboratif qui permet la création d'un lien social fort avec l'entrepreneur. Le contrat est détourné de sa finalité première pour être utilisé comme une modalité d'encastrement social.

\section{BibLIOGRAPHIE}

Burt R., Structural Holes, Harvard University Press, 1992.

Bygrave W.D., "Syndicated investments by venture capital firms: a networking perspective", Journal of Business Venturing, 2, 1987, p. 139-154.

Ferrary M., "The gift exchange in the Social Networks of Silicon Valley", California Management Review, vol. 45, n 4, 2003, p. 120-138.

Ferrary M., Pesqueux Y., L'organisation en réseau. Mythes et réalités, PUF, 2004.

Gompers P., Lerner J., "Venture capital distributions: short-run and long run reactions", The Journal of Finance, vol. LIII, n 6, 1998, p. 2161-2 183.

Gompers P., Lerner L., "The Venture Capital Revolution", Journal of Economic Perspectives, vol. $15, \mathrm{n}^{\circ} 2,2001$, p. 145-169.

Granovetter M., "The Strength of weak ties", American Journal of Sociology, n 78, 1973, p. $1360-1380$.

Granovetter M., "The Impact of Social Structure on Economic Outcomes", The Journal of Economic Perspectives, vol. 19, n 1, 2005, p. 33-50.

Knight F., Risk, Uncertainty and Profit, Houghton Miffin Company, 1921, 375 p.

Mauss M., «Essai sur le don», Année Sociologique, t. 1, 1930.

Nonaka I., "A Dynamic Theory of Organizational Knowledge Creation", Organization Science, vol. 5, $\mathrm{n}^{\circ} 1,1994$, p. 14-38.

Penrose E., The Theory of the Growth of the Firm, Oxford, Basil Blackwell, 1959.

Tsai W., "Social capital, strategic relatedness, and the formation of intra-organizational linkages", Strategic Management Journal, n 21, 2000, p. 925-993. 